\title{
THE PROJECT OSI
}

\author{
M. SHAO
}

Abstract. The OSI project at JPL is a study of a series of concepts for space based astrometric interferometry. The various concepts are designed for slightly different types of astrometry. The original concept OSI was designed for wide angle/global astrometry by look at stars about 30 degrees apart. A derivative of OSI, called SONATA was designed for narrow angle astrometry (10 arcmin) at sub-microarcsec levels for exo-planet searches. This paper describes optical concepts of theses instruments with regard to metrology and systematic error. In addition to conceptual designs, system error budgets and covariance analysis the OSI project has a technology development program in place to investigate the most difficult problems.

Technology is being persued in three areas. One is laser metrology at the picometer level where sub-picometer accuracy for null metrology has been achieved and $<2$ picometer accuracy has been achieved for relative metrology. A second area is the control of vibration on a large space structure. A $5 \mathrm{~m}$ truss structure has been built at JPL with an interferometer on the structure to test vibration isolation and control technologies. Tests with this structure will begin in winter of 1994 . The key to vibration control for OSI will be the delay lines. The third technology project is a shuttle flight experiment, Stellar Interferometer Technology Experiment (SITE), currently in a phase A study, (part of NASA's INSTEP program). The goal is to fly the experiment on the Shuttle in 1997-1998. 